\title{
Etude physico-chimique des sédiments du Lac de St-Point (massif du Jura) : cartographie thématique ${ }^{1}$
}

\author{
J. Verneaux ${ }^{2}$ \\ A. Guyard2 \\ D. Joly ${ }^{3}$ \\ A. Vidonne ${ }^{4}$
}

Mots clés : sédiments lacustres, paramètres physico-chimiques, cartographie, fonctionnement.

Les analyses physico-chimiques portent sur 180 carottes sédimentaires réparties en 30 stations jalonnant la cuvette lacust re. Les variations, verticales (enfoncement dans le sediment) et longitudinales, des descripteurs majeurs sont analysées ; puis, six représentations cartographiques (logiciel DIGICART) rendent compte de leurs distributions spatiales et soulignent les principales tendances fonctionnelles du système, plus particulièrement le rôle du Doubs, vecteur de matériaux allochtones et de nutriments.

Physical and chemical studies on the sediments of the St-Point lake (Jura mountains)

Keywords: lake sediments, physical and chemical parameters, mathematical cartography, dynamic.

Physical and chemical analyses were made on 180 sediment cores, taken at 30 sites of the lake bottom area, at several depths.

The horizontal and vertical changes of the major parameters are analysed; then six statistical maps show their spatial distributions and the outlines of the sedimentation processes. The influence of the Doubs river, as a vector of nutrients and other allochtonous materials, is specially emphasised.

\section{Situation et caractères généraux du lac de Saint-Point}

Ce chapitre introductif constitue le sommaire d'une monographie (Univ. Fr, Comté, 1979) ayant constitué le premier élément d'une série d'études collectives (S.R.A.E. Fr. Comté - C.E.M.A.G.R.E.F.,

1. Recherche bénéficiant d'un Contrat de Plan Etat-Région (S.R.E.T.I.E. - Ministère de l'Environnement et d'un soutien de la Direction à ta Recherche de l'Enseignement Supérieur).

2. Laboratoire d'Hydrobiologie - Hydroécologie, Faculté des Sciences Naturelles ; Place Leclerc, 25030 Besançon Cedex, France.

3. Laboratoire de Géographie physique. Faculté des Lettres et Sciences Humaines. Rue Mégevand, 25030 Besançon Cedex. France.

4. Laboratoire de Chimie des Eaux, Faculté des Sciences, Rue Mégevand, 25030 Besançon Cedex, France.

Lyon - Univ. Fr. Comté) consacrées aux lacs du Jura franc-comtois et publiées sous l'égide de l'Etablissement Public Régional (E.P.R. Fr. Comté, 1982-1987).

Des données figurent également dans des travaux plus anciens comme le traité général de Delebecque (1898), l'ouvrage de Magnin (1904) sur les macrophytes ou l'étude à caractère appliqué réalisée par Kreitman (1937) alors que le lac était encore utilisé industriellement.

Le plus vaste des lacs naturels du Jura franccomtois s'inscrit, à $850 \mathrm{~m}$ d'altitude, dans le paysage de la haute vallée du Doubs, dominé par les pâturages et les sapinières.

Dans un climat marqué à la fois par une grande amplitude thermique et par une forte pluviosité (environ $1500 \mathrm{~mm}$ d'eau par an dont $30 \%$ de neige), 


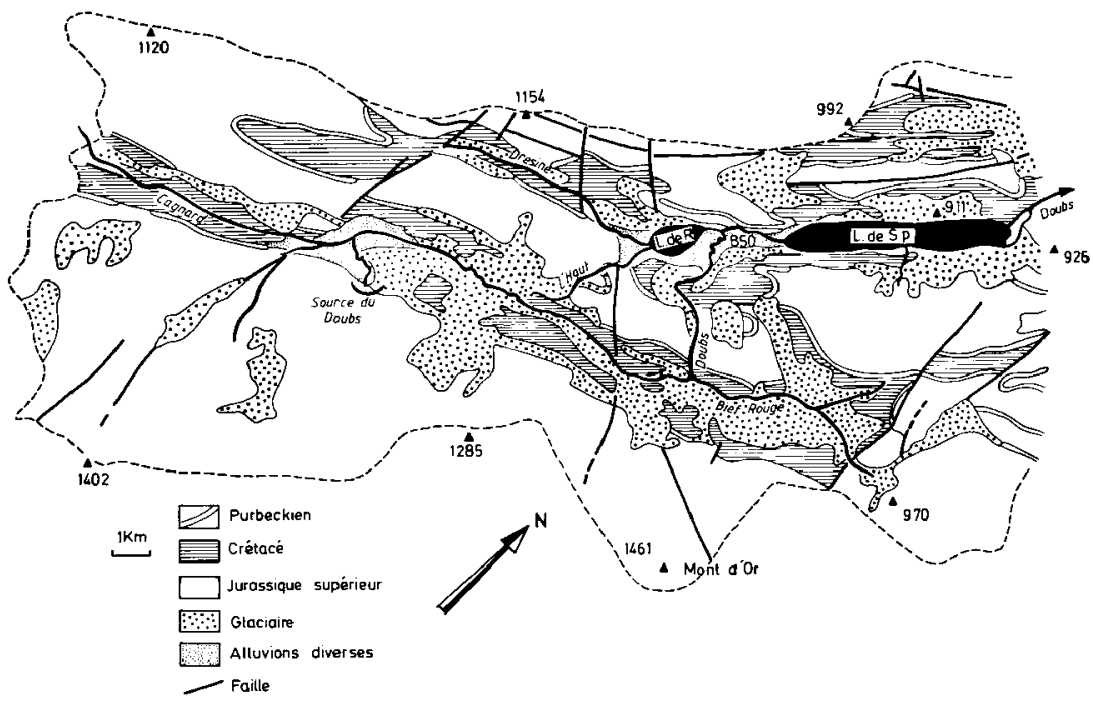

Fig. 1. Géologie du bassin-versant du lac de St Point et réseau hydrographique

la surface du lac est prise en glace durant 4 à 5 mois mais peut, en juillet-août, at teindre des températures voisines de $23^{\circ} \mathrm{C}$.

L'économie traditionnelle, basée sur l'exploitation forestière, l'élevage et les industries laitières, se complète des activités associées au tourisme et aux sports d'hiver, plus particulièrement dans la partie apicale de la vallée du Doubs, et de son affluent, le Bief Rouge, qui constituent actuellement les principaux vecteurs de pollution des eaux, depuis la mise en place en 1980 d'un collecteur des eaux usées à la périphérie du lac.

Le lac, traversé par le Doubs, occupe une dépression synclinale où les formations du Crétacé (AlboCénomanien) sont surmontées de placages glaciaires (fig. 1) ; à la fonte du glacier würmien, les moraines ont assuré la fermeture de la cuvette en créant un unique plan d'eau; les lacs de St Point et de Remoray ont été séparés ultérieurement par accumulation des alluvions du Doubs.
Les eaux, alcalines, bien minéralisées, enrichies des composés azotés et phosphorés issus du Doubs, induisent une forte production primaire ; les ceintures végétales (phragmito-scirpaie, potamaie, nupharaie + Hippuris et Characées) sont favorables à la reproduction des Percidés et Cyprinidés ainsi qu'au développement des stades juvéniles et à l'hébergement des Brochets. Le peuplement pisciaire dense, comprenant 10 especes recensées, est dominé par le Corégone (Palée), la Perche et le Gardon. Les caractéristiques morphométriques et physicochimiques générales figurent dans le Tableau I.

\section{COMPLEMENTS RELATIFS A L'OXYGENATION DES EAUX PROFONDES}

Une série de mesures réalisée en novembre 1985 , avant la circulation préhivernale intéressant toute la tranche d'eau (lac holomictique), a mis en évidence l'hétérogénéité de la répartition des concentrations en oxygène dissous de la couche d'eau 
Tableau I. Caractéristiques générales du lac de St Point (univ. Fr. Comté, 1979 . Blanc \& Beyne 1986)

\begin{tabular}{|c|c|c|c|c|c|c|}
\hline$L \min _{(m)}(\ell)$ & $\begin{array}{l}\varepsilon \max (b) \\
(\mathrm{m})\end{array}$ & $\underset{(m)}{\text { périm. (L) }}$ & $\begin{array}{l}S(A) \\
(1, n)\end{array}$ & $\left(10^{6} \mathrm{~m}^{3}\right)$ & $\begin{array}{c}\mathrm{BV} \\
\left(\mathrm{km}^{2}\right)\end{array}$ & (m) \\
\hline 6250 & 840 & 14200 & 419 & 84 & 241 & 850 \\
\hline $\begin{array}{l}\text { prof. max. } \\
\text { (z) }\end{array}$ & $\begin{array}{l}\text { prof. moy. } \\
\text { (z) }\end{array}$ & $\begin{array}{c}Q \text { m.a. Doubs } \\
\left(m^{\prime} / s\right)\end{array}$ & $\begin{array}{c}\bar{E} \text { renouvelt } \\
(j / a n)\end{array}$ & $\begin{array}{r}\text { pompage } \\
\left(m^{3} / j\right)\end{array}$ & $\begin{array}{c}\text { transparence } \\
\text { disque : } 5\end{array}$ & $\begin{array}{c}c \\
(\mu S / c m)\end{array}$ \\
\hline 40 & 20.3 & 4 & 200 & 21000 & $\begin{array}{l}\max : 9 \\
\operatorname{mini}: 1.8 \\
\text { (vIII) }\end{array}$ & $\begin{array}{l}\max : 340 \\
\operatorname{mini}: 250\end{array}$ \\
\hline $\mathrm{pH}$ & $\begin{array}{l}\text { alcalinité } \\
\left(\mathrm{mg} / \mathrm{P} \mathrm{HCO}_{3}^{-}\right)\end{array}$ & $\begin{array}{l}\text { Nitrates } \\
\left(\mathrm{me} /\left(\mathrm{NO}_{3}^{-}\right)\right.\end{array}$ & $\begin{array}{l}\text { Phosphates } \\
\left(\mu g / \mathrm{FO}_{4}^{--} \text {) }\right.\end{array}$ & $\begin{array}{l}{ }^{14} \mathrm{C} \\
\left(\mathrm{mg} \mathrm{c} \text { ass } / \mathrm{m}^{2} / \mathrm{j}\right.\end{array}$ & $\begin{array}{l}\operatorname{chl} a \\
(\mu, / C)\end{array}$ & $\begin{array}{l}\text { tempé } r \text { ature } \\
\text { max. } \\
\left({ }^{\circ} \mathrm{C}\right)\end{array}$ \\
\hline $\max .: 8.7$ & $\max .: 250$ & $\max .: 3.10$ & $\max -:=170$ & $\max .: 661$ & $\min x .: 28$ & surface $=22.5$ \\
\hline $\operatorname{mini}: 7.4$ & mini: 195 & mini: 0.05 & $\operatorname{mini}: 52$ & (VIII) & (VIII) & fond $=8.1$ \\
\hline
\end{tabular}

Tableau II. Oxygénation et potentiel redox des eaux de contact en $\therefore$. rofondeurs différentes - 8 mesures/profondeur - 8/10/85

\begin{tabular}{|c|c|c|c|c|c|c|}
\hline \multirow{2}{*}{$\begin{array}{l}\text { Prof. } \\
\text { fond }(m)\end{array}$} & \multicolumn{3}{|c|}{$\mathrm{O}_{2}(\mathrm{mg} / \mathrm{l})$} & \multicolumn{3}{|c|}{$\pi$ sat. } \\
\hline & $\max$ & $\bar{n}$ & $\operatorname{mini}$ & $\max$ & $\bar{m}$ & $\operatorname{mini}$ \\
\hline-10 & 6.1 & 4.6 & 3.5 & 54.3 & 43.0 & 33.0 \\
\hline-20 & 3.4 & 3.3 & 2.6 & 29.3 & 28.8 & 23.0 \\
\hline-30 & 2.3 & 1.4 & 0.8 & 19.3 & 11.9 & 5.8 \\
\hline-35 à -39 & 1.5 & 0.9 & 0.6 & 11.0 & 8.0 & 5.0 \\
\hline
\end{tabular}

en contacl avec les sédiments (eaux de contact Tableau II).

Les mesures effectuées in situ à $10 \mathrm{~cm}$ du fond à l'aide d'un oxymètre ont été contrôlées par prise d'un échantillon par station, fixé sur place pour un dosage par la méthode de Winckler.

On observe que les concentrations indiquées sont très inférieures à celles mentionnées par Kreitman (77\% de saturation à $-39 \mathrm{~m}$ en Novembre 1933) ainsi qu'à celles relevées en Novembre 1978 (3.2 mg/l à - $35 \mathrm{~m}$ ). On note également que les valeurs diffèrent sensiblement d'un point à l'autre; les influences du Doubs et d'éventuelles sources immergées ont déjà été évoquées par Kreitmann (op. cit.).

\begin{tabular}{|c|c|c|c|}
\hline $\mathbf{E}$ & \multicolumn{2}{|c|}{$\left(\mathrm{mv} / \mathrm{AgCl}_{\mathrm{C}}\right)$} & \multirow[t]{2}{*}{$\overline{\mathbf{r H}}$} \\
\hline $\max$. & $\bar{\pi}$ & $\operatorname{mini}$ & \\
\hline-192 & +136 & +66 & 29.9 \\
\hline+200 & +121 & +76 & 26.8 \\
\hline-30 & -70 & -90 & 19.9 \\
\hline-65 & -128 & -170 & 17.8 \\
\hline
\end{tabular}

Des taux de saturation inférieurs à $20 \%$ ont été relevés au niveau de la couche d'eau profonde (dernier mètre) durant une période relativement courte (fin Septembre à Novembre sans toutefois que s'instaurent des conditions réductrices durables.

\section{Les sédiments}

\subsection{Nature des recherches et méthodes}

Dans une publication récente présentant les caractéristiques physico-chimiques générales des sédiments de 10 lacs francs-comtois, (Verneaux et al. 1987) chaque lac était représenté par un échantillon 


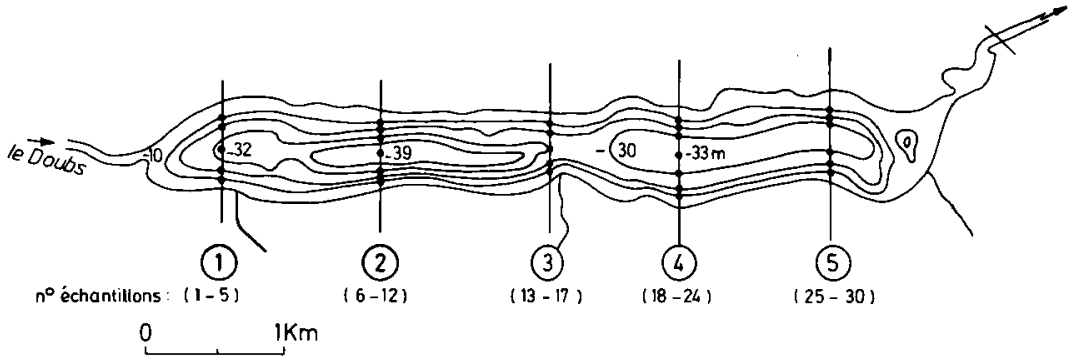

Fig. 2. Bathymétrie du lac de St Point et plan d'échantillonnage -9.11/10/85

sédimentaire moyen (4 prélèvements de la couche superficielle $0-2,5 \mathrm{~cm}$ ) obtenu par carottage au point le plus profond de chaque lac.

Dans un premier classement des échantillons sédimentaires, selon la perte au feu (PF), assimilable à la matière organique, et la teneur en carbonates ou d'après le carbone organique et le rapport $\mathrm{Ca} / \mathrm{Si}$, l'échantillon du lac de St Point se situe en troisième position dans une gamme allant du sédiment profond le plus clair ( $\mathrm{PF}<10 \%, \mathrm{CaCO}_{3}>75 \%$ - lac de Clairvaux) au sédiment profond le plus sombre (PF $>25 \%, \mathrm{CaCO}_{3}<25 \%$, lacs de l'Abbaye et Bonlieu).

Après que des études préalables aient mis en évidence la très grande variabilité des échantillons littoraux, soumis à diverses influences ponctuelles (C.E.M.A.G.R.E.F, in E.P.R. Fr. Comté 1985, Jarrot 1983, Lagadec \& Thoumy 1984, Bernard \& Deroletz 1985), les travaux furent centrés sur l'analyse des variations horizontales et verticales de la composition sédimentaire en zone profonde (de - 10 à - $40 \mathrm{~m}$ ) et de la surface du sédiment à $30 \mathrm{~cm}$ (enfoncement).

A défaut de pouvoir réaliser une prospection systématique selon un quadrillage régulier à maiłle serrée (Cahill 1981) il a été choisi de définir un protocole qui permette :

- d'une part, la réalisation d'une cartographie exprimant la distribution spatiale des principales variables,

- d'autre part de fournir des informations permettant de vérifier les hypothèses précédemment émises (Verneaux et al. op. cit.) sur l'origine des matériaux sédimentés et de guider la définition d'un protocole réduit d'échantillonnage susceptible d'application plus genérale.

\section{CARTOGRAPHie}

Parmi les nombreuses techniques permettant l'interpolation des relevés ponctuels à l'espace géographique dans son continuum, nous avons choisi d'utiliser celle des « surfaces de tendance " (in Ciceri et al. 1977).

L'application de cette technique qui utilise l'algorithme des régressions multiples appliqué à l'espace, a été exposée antérieurement (Brossard et al. 1986-1987): si, théoriquement, un polynome peut être développé jusqu'au degré N-1, N étant le nombre de stations, il convient cependant d'éviter de dépasser les degrés 6 et 7 . Les cartes présentées (fig. 9 à (1) sont issues d'un calcul arrêté au degré 4 pour lequel nous avons obtenu des valeurs satisfaisantes $(0,81<R \leqslant 0,93)$ du coefficient de corrélation multiple (Rx.yz in Spiegel 1982).

Le document cartographique constitue une synthèse des informations et un modèle de la distribution spatiale des phénomènes mis en évidence. Les cartes ont été réalisées à l'aide du logiciel Digicart (Brossard et al. 1986).

\subsection{L'échantillonnage}

Les stations sont réparties sur cinq transects sensiblement distants de $1 \mathrm{~km}$ (fig. 2) et placées, sur 


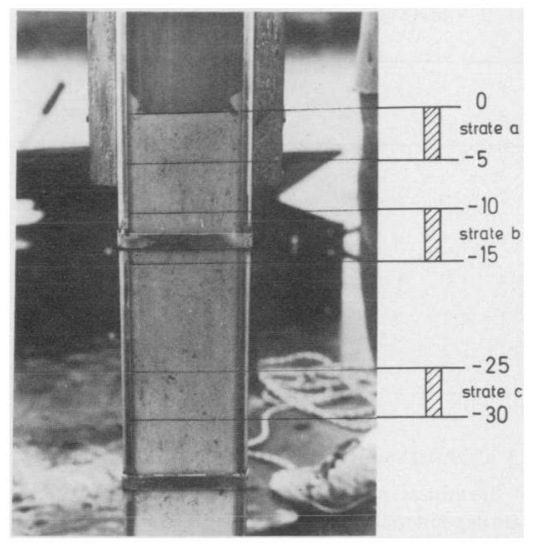

Fig. 3. Carotte sédimentaire remontée sur le ponton par la trappe centrale - St Point : - $33 \mathrm{~m}$ - strates prélevées. On observe la teinte ocrée due aux composés ferrugineux.

chacun d'entre eux, aux profondeurs de $-10,-20,-30$ et $-40 \mathrm{~m}$ (ou fond). Les prélèvements sont effectués depuis un ponton automoteur à treuil central, à l'aide d'un carottier à paroi transparente coulissante (Rofes \& Savary 1981) permettant l'obtention d'une carotte non modifiée; deux prélèvements de $100 \mathrm{~cm}^{2} \times 5 \mathrm{~cm}$ sont réalisés par station afin d'ob. tenir un échantillon moyen de $1 \mathrm{dm}^{3}$ pour chaque strate $(0-5 \mathrm{~cm}, 10-15 \mathrm{~cm}$ et $25-30 \mathrm{~cm}$ - photographie fig. 3).

Les analyses portent donc sur 90 échantillons issus de 180 prélèvements réalisés sur 30 stations.

Nota : deux prélèvements contenant des Characées vivaces ont été rejetés.

Conjointement, les paramètres suivants sont mesurés sur les eaux de contact : $\mathrm{pH}, \mathrm{Eh}$, conductivité électrique, température et oxygène dissous ; un échantillon de $1 \mathrm{l}$ est également prélevé aux fins d'analyses complémentaires dont les résultats seront présentés ultérieurement.

Lès échantillons sédimentaires sont placés immédiatement en enceinte réfrigérée à $4^{\circ} \mathrm{C}$ dans des récipients hermétiques indéformables.
Après séchage à l'étuve à $85^{\circ} \mathrm{C}$, jusqu'à poids constant, les échantillons broyés et homogénéisés sont soumis à analyse le plus rapidement possible.

\section{Résultats}

\subsection{Composition chimique générale}

\subsubsection{DESCRipteurs}

Les valeurs figurant dans le tableau III représentent des pourcentages de matière sèche, à l'exception de la teneur en eau (t.e.h.) et de l'indice de réflectance (Ir) exprimant la teinte du sédiment, d'autant plus foncée que celui-ci est riche en matière organique (Verneaux et al. 1987, Vidonne et al. 1987).

Les méthodes d'analyse ont été indiquées dans ces dernières publications.

Les carbonates $(76 \%$ m.s. relativement à l'échantillon global et $68 \%$ dans la couche superficielle en zone profonde) représentent le principal constituant de cet te " craie lacustre " formée par précipitation chimique.

On observe par ailleurs, que dans ce lac à forte productivité primaire $(\mathrm{Ch} l \mathrm{a}$. $\max >70 \mu \mathrm{g} / l$. Barbe in Univ. Fr. Comté 1979), la teneur en matière organique résiduelle (non recyclée ou diffusée sous forme minérale assimilable) est sensiblement moitié moindre (7\% m.s.) que dans le lac Pavin (16\% m.s., Restituito 1984), cependant beaucoup moins productif (Chl a. $\max .<20 \mu \mathrm{g} / l$, Restituito \& Lair : 1975-1976). On peut penser que ce dernier système, méromictique et anoxique, de façon durable, de - 60 à - $90 \mathrm{~m}$, ne présente pas, actuellement et contrairement au lac de St Point, un mode de fonctionnement susceptible de s'inscrire dans un véritable processus d'eutrophisation dans la mesure où il minéralise très partiellement la faible quantité de matière organique autochtone ou issue de son bassin-versant. Les données relatives à la production phytoplanctonique du lac de St Point sont toutefois à actualiser, compte-tenu des travaux d'épuration réalisés depuis 1979 .

\subsubsection{VARIABILITÉ PHYSICO-CHIMIQUE GÉNÉRALE SELON LES PROFONDEURS ET LES STRATES}

Tracée à partir des valeurs figurant dans des tableaux identiques au suivant (Tableau III), établis pour chaque strate $(a, b, c)$ et par profondeur, la figure 4 représente la distribution du coefficient de 
Tableau III. Composition chimique de l'échantillon sédimentaire global variables et paramètres de distribution -84 individus - $\mathrm{PF}=$ perte au feu, $\mathrm{Co}=$ carbone organique

\begin{tabular}{|c|c|c|c|c|c|c|c|c|c|c|c|c|c|c|}
\hline$n=84$ & $\mathbf{k}$ & t.e.h. & PE & co & NK & $C / N$ & $\begin{array}{c}\mathrm{CO}_{3} \\
\left(\mathrm{r}_{\mathrm{a}} \mathrm{CO}_{3}\right)\end{array}$ & $\mathrm{Ca}$ & si & $\mathrm{Ca} / \mathrm{Si}$ & $\left(\mathrm{PO}^{3-}\right)$ & $\begin{array}{l}\mathrm{Fe}_{2} \\
\left(\mathrm{Fe}_{2} \mathrm{n}_{3}\right)\end{array}$ & $\begin{array}{c}\mathrm{Mn} \\
\left(\mathrm{WnO}_{\underline{7}}\right)\end{array}$ & $\begin{array}{c}k \\
(k, 0)\end{array}$ \\
\hline $\min$ & 27.0 & 47.0 & 2.38 & 1.02 & 0.09 & 8.514 & 35.1 & $\because 4,1$ & 0.95 & 2,53 & 0.017 & $0.2 \div 9$ & 0.013 & $0.0 \mathrm{~B}$ \\
\hline $\bar{x}$ & 43.7 & 65.6 & 6.77 & 2.89 & 0.29 & 10.24 & 75.0 & 31,2 & 15.79 & 22.68 & 0.218 & 1.377 & 0.035 & 0.207 \\
\hline $\max$ & 70.0 & 80.0 & 11.67 & 5.05 & 0.55 & 13.59 & 97.5 & 38,8 & 9.77 & 40,84 & 0.611 & 2.487 & 0.185 & 0.443 \\
\hline$\sigma^{2}$ & 83.9 & 58.9 & 5.44 & 1.05 & 0.14 & 1,50 & 72.9 & 9.9 & 116.80 & 261.05 & 0.018 & 0.283 & 0.001 & $0.0 \mathrm{nn}$ \\
\hline$\sigma$ & 9.1 & 7.7 & 2.33 & 1.02 & 0.12 & 1.22 & B. 5 & 3.1 & 10.71 & 16.16 & 0.135 & 0.532 & 0.026 & 0.095 \\
\hline$v=\frac{0}{x}$ & 0.2 & 0.1 & 0.34 & 0.35 & 0.41 & 0.12 & 0.1 & 0.1 & 0.67 & 0.7 & 0.619 & 0.386 & 0.481 & 0.458 \\
\hline
\end{tabular}

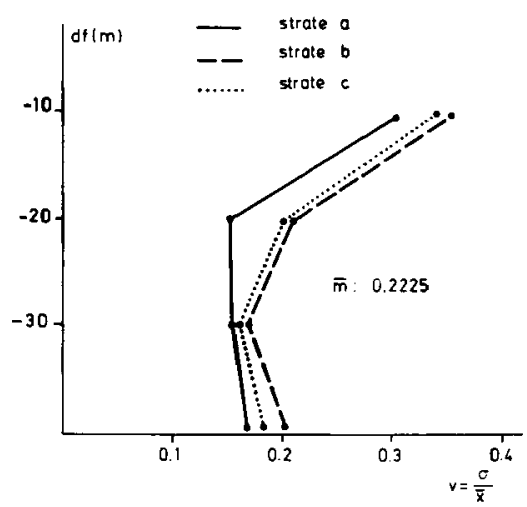

Fig. 4. Variabilité générale des valeurs paramétriques aux différentes profondeurs pour les différentes strates.

variation $\left(v=\frac{\sigma}{\bar{x}}\right)$ aux différentes profondeurs et
par strate.

Quelle que soit la strate considérée, l'homogénéité du sédiment s'affirme au-delà du talus, le niveau . $10 \mathrm{~m}$ étant encore soumis à l'influence du matériel littoral qui s'accumule au pied du mont (Verneaux et al. op. cit.).

Les écarts ne paraissent pas importants d'une strate à l'autre et son particulièrement faibles à - $30 \mathrm{~m}$ (plaine).

\subsubsection{ANALYSE DES RELATIONS ENTRE VARIABLES}

Le tableau suivant donne les valeurs du coefficient de dépendance de Pearson $(-1 \leqslant r \leqslant 1)$ appliqué à l'ensemble des 14 variables.

Ces résultats confirment les relations générales mises en évidence antérieurement par l'analyse des données issues de dix lacs jurassiens (Verneaux et al. op. cit.) ; l'an tagonisme de deux groupes de paramètres covariants domine la composition sédimentaire :

- celui des carbonates, corrélé positivement avec l'indice de réflexion,

- celui de la matière organique et ses constituants principaux, associé, avec une moindre intensité, à la Silice.

On observe que si la très forte corrélation entre la perte au feu et l'Azote Kjeldahl confirme la nature essentiellement organique de l'Azote des sédiments, il est en revanche probable qu'une proportion non négligeable du Phosphore soit imputable à des phosphates minéraux "piégés".

Contrairement aux résultats obtenus au lac Pavin (Restituito 1984), le Fer n'est corrélé ni avec la matière organique. ni avec le rapport $\mathrm{C} / \mathrm{N}$ dont l'indépendance générale a déjà été soulignée (Verneaux et al. op. cit.). L'intensité des relations entre la matière organique et le Fer est susceptible de présenter de grandes variations d'un lac à l'autre du fait que ce dernier élément peut se combiner avec les carbonates (Sidérite), les silicates, les phosphates, les sulfates... ainsi qu'avec la matière organique dans des complexes ferro-humiques (Jenne 1977 - 
Tableau IV. Matrice des relations interparamétriques - coefficient de dépendance de Pearson - 84 individus $\mathrm{PF}=$ perte au feu, $\mathrm{Co}=$ carbone organique.

\begin{tabular}{|c|c|c|c|c|c|c|c|c|c|c|c|c|c|c|}
\hline Ir & c.c.h. & : & $C o$ & $\mathrm{NK}$ & $C / N$ & $m_{3}$ & Ca & $5 i$ & $\int a / s i$ & $\mathrm{Ft}$ & Ior & 9113 & $k$ & \\
\hline \multirow[t]{14}{*}{1} & -0.39 & -0.70 & -0.75 & -0.73 & -0.03 & -0.76 & -0.74 & -0.76 & -0.74 & $-n . t .1$ & $-0,4 \pi$ & -0.23 & -0.36 & Ir \\
\hline & & $0.6 n$ & 0.52 & 0.55 & -0.11 & -0.48 & -0.52 & 0.38 & -0.49 & 0.3 .3 & n. 12 & -0.15 & 0.50 & t.e.h \\
\hline & & & 0.91 & 0.89 & 0.09 & 0.79 & -0.83 & -0.66 & 0.63 & -0.64 & 0.42 & n.od & 0.67 & $\mathbf{P F}$ \\
\hline & & & & 0.96 & 0.11 & -0.82 & -0.81 & 0.70 & -0.60 & 0.6 .3 & 0.34 & -0.11 & $0.01,3$ & $c a$ \\
\hline & & & & & 0.13 & -0.75 & -0.75 & 0.67 & -0.58 & 0.62 & 0.28 & -0.15 & 0.56 & NK \\
\hline & & & & & & $-0,24$ & -0.25 & 0.09 & -0.0 .5 & 0.06 & 0.26 & 0.14 & 0.28 & $\mathrm{C} / \mathrm{N}$ \\
\hline & & & & & & & 0.89 & -0.77 & 0.70 & -0.6 .1 & $-n .46$ & -0.00 & -0.70 & $\mathrm{CO}_{3}$ \\
\hline & & & & & & & & -0.78 & 0.74 & -0.64 & -0.50 & -0.13 & -0.77 & $\mathrm{Ca}$ \\
\hline & & & & & & & & & -0.79 & 0.50 & 0.42 & $0.1 \%$ & 0.52 & Si \\
\hline & & & & & & & & & & -0.57 & -0.33 & -0.32 & -0.50 & $\mathrm{Ca} / \mathrm{Si}$ \\
\hline & & & & & & & & & & & 0.38 & 0.24 & 0.45 & Ft \\
\hline & & & & & . & & & & & & & n. .38 & 0.47 & $\mathrm{Fe}$ \\
\hline & & & & & & & & & & & & & -1.06 & in: \\
\hline & & & & & & & & & & & & & & $\mathrm{K}$ \\
\hline
\end{tabular}

auct. in Hakanson \& Jansson 1983, Tipping \& Ohnstad 1984).

Une indication supplémentaire apparait : la relation assez nettement négative s'établissant entre Calcium et Potassium $(\cdot 0,77)$, vraisemblablement due au fait que ce dernier élément proviendrait, en grande partie, de l'Illite des argiles allochtones.

\subsection{Vartations verticales des deseripteurs majeurs}

\subsubsection{VARIATIONS DE MO ET DU RAPPORT Ca/Si}

Suivant une observation usuelle dans le cas des lacs peu profonds, le pourcentage de matière organique augmente avec la profondeur, environ d'un tiers entre $\cdot 10$ et $\cdot 40 \mathrm{~m}$ et décroît assez régulièrement avec l'enfoncement dans le sédiment, d'environ $20 \%$ dans les premiers 20 centimètres soit de façon approchée de $1 \%$ par cm (fig. 5A).

L'hypothèse la plus vraisemblable est que, dans la strate superficielle, une fraction de l'Azote organique provient du matériel biologique à faibles rapports $\mathrm{C} / \mathrm{N}$ (généralement compris entre 4 et 8 , Domergues \& Mancenot 1970, Vinogradov 1953, Lehninger $1970 \ldots)$, qu'il s'agisse du macrobenthos de cette zone de bioturbation (Oligochètes, Diptères Chironomidés, Pisidies...), des Protozoaires ou de la

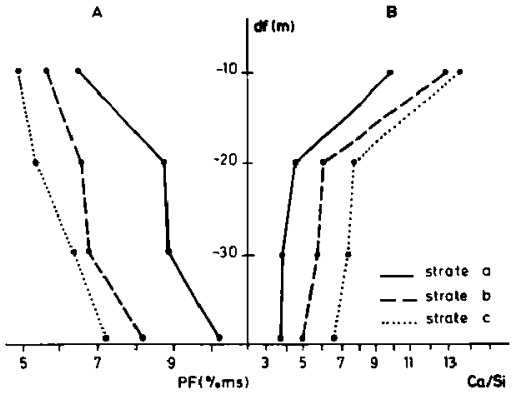

Fig. 5. Variations inverses du pourcentage de matière organique (PF, A) et du rapport $\mathrm{Ca} / \mathrm{Si}$ (B) selon la profondeur et les strates $-\overline{\mathbf{m}} 10$ à 4 échantillons par point.

biomasse bactérienne intervenant dans la transformation de la matière organique.

Cette interprétation a été proposée par Kleerekoper (1957) : l'augmentation du rapport $C / N$ est associée à une diminution de l'activité bactérienne et, conjointement, de la vitesse de transformation de la 


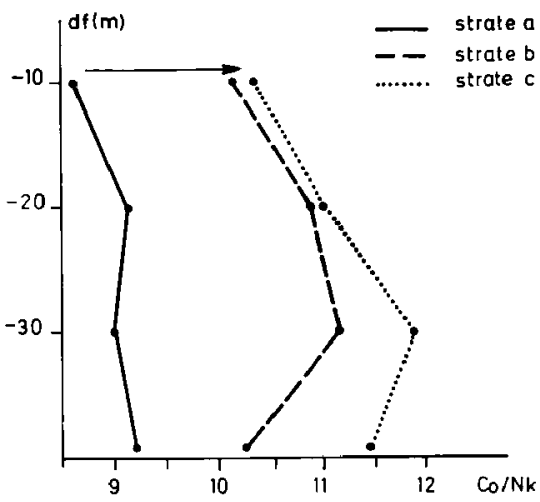

Fig. 6. Variation du rapport $\mathrm{C} / \mathrm{N}$ selon la profondeur et les strates $-\overline{\mathrm{m}} 10$ à 4 échantillons par point.

MO qui se trouverait ainsi, sinon stabilisée, du moins beaucoup plus lentement mobilisée.

Ces données concordent avec le point de vue de Kemp (1971) associant l'augmentation du C/N à une dégradation des complexes protéiques puis à une diffusion des formes minérales dans les eaux de contact.

La détermination des formes de la fraction conservée de matière organique est à inclure dans un protocole plus complet d'analyse comparée.

Cette évolution vers la craie lacustre correspond à une augmentation du même ordre de grandeur ( $20 \%$ de la strate a à la strate c) des carbonates et à une diminution conjointe du Silicium de l'ordre de 30 à $40 \%$.

Le rapport $\mathrm{Ca} / \mathrm{Si}$ augmente donc suivant les strates, d'environ $1 / 3$ sur les 25 premiers centimètres, mais décroit inversement à la matière organique (relation MO-Si $=+0.66$ ) selon la profondeur ( $f i g$. $5 \mathrm{~B})$.

\subsubsection{Evolution de l'azote, du Carbone organique ET DU RAPPORT $\mathrm{C} / \mathrm{N}$}

La minéralisation du sédiment s'accompagne d'une augmentation du rapport $\mathrm{C} / \mathrm{N}$, légèrement selon la profondeur jusqu'à - $30 \mathrm{~m}$, beaucoup plus nettement selon l'enfoncement dans le sédiment ( $f i g .6)$.
Le même phénomène est mentionné par Restituito (1984) pour les sédiments du lac Pavin, ainsi que le fait d'une tendance à la stabilisation ou à l'inversion des variations à partir de $-30 \mathrm{~m}$.

Le tableau $V$ et la figure 7 mettent en évidence le fait que l'augmentation du rapport $\mathrm{C} / \mathrm{N}$ correspond à un déficit azoté plus important que celui du carbone, l'écart se situant entre 10 et $15 \%$.

Ce phénomène est actuellement analysé par la prise en compte des différentes fractions granulométriques.

\subsubsection{DISTRIBUTION LONGITUDINALE}

L'examen des variations longitudinales de la matière organique (PF) et du rapport $\mathrm{C} / \mathrm{N}$ (fig. 8) de la couche superficielle $(0-5 \mathrm{~cm})$ s'accorde avec l'hypothèse précédemment émise, en mont rant que si, transversalement la proportion en MO et le rapport $\mathrm{C} / \mathrm{N}$ augmentent du mont vers la plaine, ces deux parametres diminuent conjointement de l'amont vers l'aval du lac; l'influence du Doubs, principal vecteur de nutriments, est ainsi mise en évidence.

Ce phénomène exprime à la fois un changement de la nature et du degré de transformation des apports organiques, de l'amont vers l'aval du lac, et de la zone sublittorale à la zone profonde.

Progressivement, la proportion de matériel organique allochtone, ou induit en tête de lac par les sels minéraux issus du Doubs, régresse en même temps que les concentrations en nutriments; dans le bassin aval du lac, le matériel planctonique autochtone devient prépondérant.

Ceci est en accord avec de nombreuses observations, réalisées au cours des campagnes d'échantillonnage du peuplement pisciaire, où des colmatages intenses des filets maillants par des Algues filamenteuses (essentiellement du genre Spirogyra) ont été régulièrement constatés en zone amont.

Le lac constitue un système épurateur des eaux issues du Doubs, contaminées par des nutriments résiduels.

\section{Cartographie des tendances}

Les résultats des précédentes analyses sont exprimés par les représentations cartographiques des figures 9 et 11 relatives aux distributions spatiales 
Tableau V. Proportions d'Azote et de Carbone organique aux différentes profondeurs, dans les différentes strates.

\begin{tabular}{|c|c|c|c|c|c|c|c|c|}
\hline & & \multicolumn{3}{|c|}{$\mathrm{CO}$} & \multicolumn{4}{|c|}{ NK } \\
\hline & & a & b & c & $a$ & $b$ & $c$ & \\
\hline $\mathrm{n}$ & $=34$ & $0-5 \mathrm{~cm}$ & $10-15 \mathrm{~cm}$ & $15-30 \mathrm{~cm}$ & $0-5 \mathrm{~cm}$ & $10-15 \mathrm{~cm}$ & $15-30$ & $\mathrm{~cm}$ \\
\hline & $\begin{array}{l}10 \mathrm{~m} \\
=\quad 24\end{array}$ & 2.97 & 2.52 & 2.23 & 0.34 & 0.24 & 0.21 & \\
\hline & $\begin{array}{l}20 \mathrm{~m} \\
=30\end{array}$ & 3.77 & 2.76 & 2.30 & 0.41 & 0.25 & 0.21 & \\
\hline & $\begin{array}{l}30 \mathrm{~m} \\
=21\end{array}$ & 3.95 & 2.70 & 2.22 & 0.44 & 0.24 & 0.18 & \\
\hline & $\begin{array}{l}40 \mathrm{~m} \\
=9\end{array}$ & 4.32 & 2.99 & 2.34 & 0.46 & 0.24 & 0.20 & \\
\hline
\end{tabular}

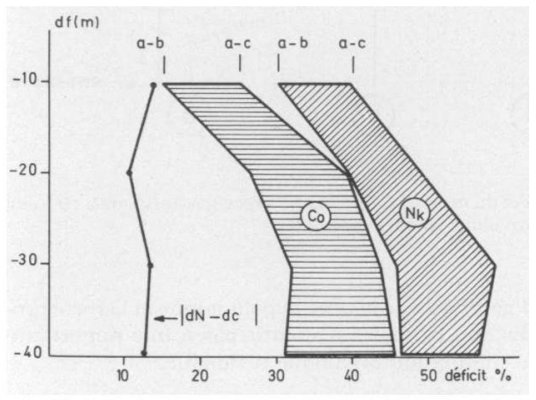

Fig. 7. Déficits comparés du Carbone organique et de l'Azote aux différentes profondeurs.

des carbonates $\left(\% \mathrm{CaCO}_{3}\right)$ de la matière organique (\% PF) et de la Silice totale $\left(\% \mathrm{SiO}_{2}\right)$ dans les strates a et c $(0-5 \mathrm{~cm}:$ A et $25-30 \mathrm{~cm}$ : B) des sédiments ; les cartes des strates b qui se sont révélées intermédiaires dans tous les cas, n'ont pas été présentées. Les processus généraux de la sédimentation dans le lac sont visualisés par la perception globale des différenciations transversales, longitudinales et verticales (dans l'épaisseur du sédiment).

La précipitation carbonatéc autochtone domine le comblement de la cuvette ( $f i g .9$ ) : elle s'effectue essentiellement dans les zones littorale et sublittorale, au contact des ceintures végétales (Portner 1951).

Dans le cas de la présence d'un vecteur fluviatile, les accumulations de craie lacustre sont de plus en plus importantes en allant vers l'aval du lac (Campy \& Richard 1985); au lac de St Point cet effet est accentué par la présence, en aval, d'une zone à faible profondeur, très riche en macrophytes, créée audelà du front morainique naturel, par la présence d'un barrage artificiel surélevant le niveau d'eau.

La concentration des carbonates en profondeur du sédiment correspond à une minéralisation active de la matière organique dans des conditions d'aérobiose dominante favorisant la diffusion des sels minéraux dans les eaux de contact (Blanc \& Beyne 1986).

Les cartes des figures 10 et 11 , très semblables, expriment les distributions de la matière organique et de la Silice totale dont les proportions, à l'inverse de celles des carbonates, croissent vers la zone centrale et régressent vers l'aval du lac ainsi que dans l'épaisseur du sédiment.

L'influence du Doubs, vecteur de matériaux allochtones (organiques et argileux) ainsi que de nutriments induisant une production végétale, en particulier benthique en zone amont, est ainsi mise en évidence. 

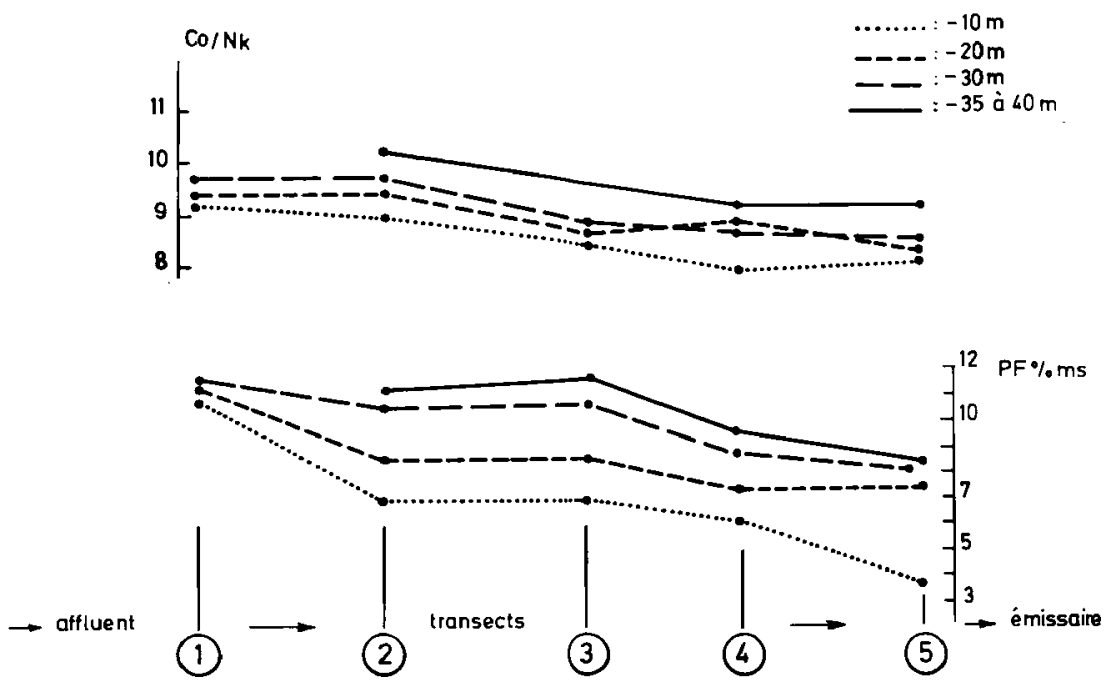

Fig. 8. Variations longitudinales conjointes du pourcentage de MO et du rapport $\mathrm{C} / \mathrm{N} \cdot$ couche superficielle (strate a : $0-5 \mathrm{~cm}$ ) aux différentes profondeurs - 28 individus - valeurs moyennes.

\section{Conclusions}

Les relations interparamétriques générales pré. sentées antérieurement (Verneaux et al. 1987) sont confirmées par la prospection méthodique réalisée qui permet d'apporter des informations complémentaires concernant la dynamique de la formation et de l'évolution du sédiment dans un cas particulier.

Dans l'exemple du lac de St Point, la précipitation carbonatée autochtone, très dominante, conduit à la formation d'une " craie lacustre ", dont la proportion en carbonates (essentiellement en $\mathrm{CaCO}_{3}$ ) croît vers l'aval du lac et selon l'enfoncement dans le sédiment. En zone profonde (-33, - 39 m) les teneurs en carbonates et en matière organique passent respectivement de 68 à $78 \% \mathrm{~m}$.s. et de 11 à $5 \% \mathrm{~m}$.s. dans les 20 premiers centimetres.

La minéralisation de la matière organique s'effectue convenablement dans des conditions dominantes d'aérobiose de la zone hypolimnique et la forte production primaire n'aboutit pas à une importante accumulation organique résiduelle.

Selon les schémas généraux proposés par Hansen (1961) puis Rohde (1969), un mode de fonctionnement qui correspondrait à des conditions \& d'eutrophie " (sensu siricto) paraît associè à des périodes d'aérobiose suffisamment longues en zone profonde et à des apports autochtones dominants. Des analyses complémentaires, relatives aux formes de la Silice et de la matière organique dans les différentes fractions granulométriques, demeurent à effectuer; elles sont inscrites dans le protocole d'analyse comparée qui est àppliqué actuellement à plusieurs lacs jalonnant la gamme proposée antérieurement (Verneaux et al., op. cit.).

Les cartes thématiques confirment deux points : d'une part, l'influence des ceintures végétales dans la précipitation carbonatée, d'autre part le róle du 

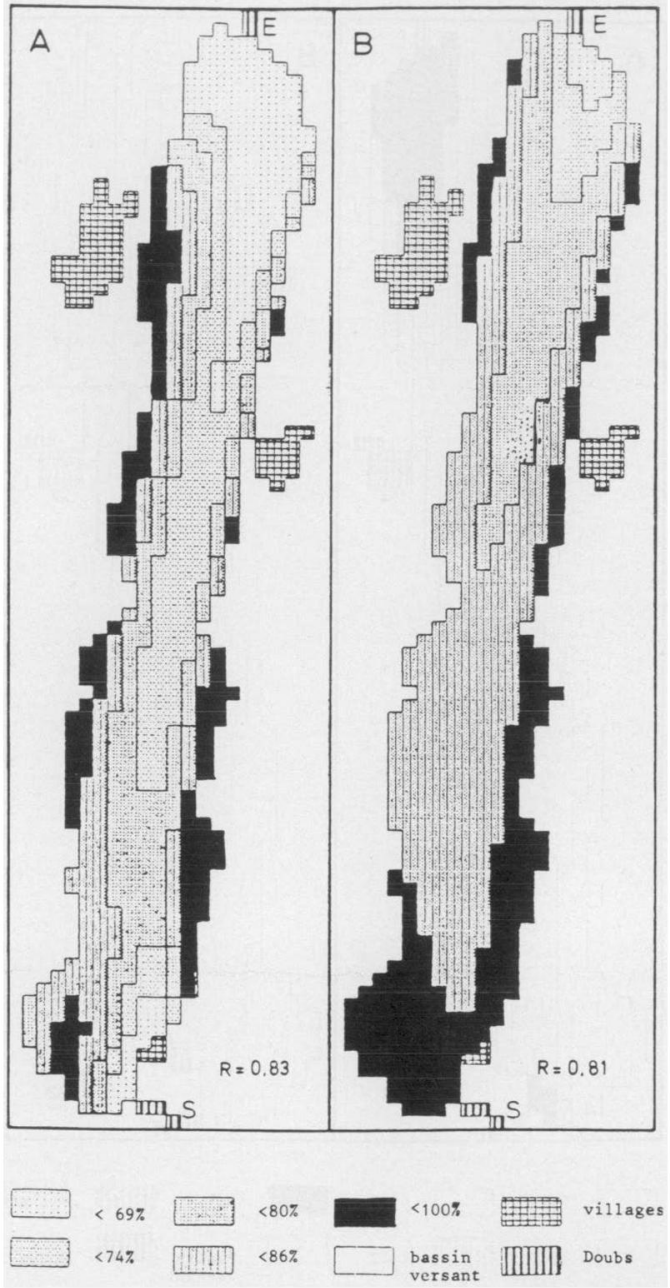

Fig. 9. Distribution spatiale des carbonates $\left(\mathrm{CaCO}_{3} \%\right.$ m.s.) dans les sédiments du lac de St Point A : strate $0.5 \mathrm{~cm}, \mathrm{~B}$ : strate $25-30 \mathrm{~cm}, \mathrm{E}$ : entrée du Doubs, $\mathrm{S}$ : sortie. 


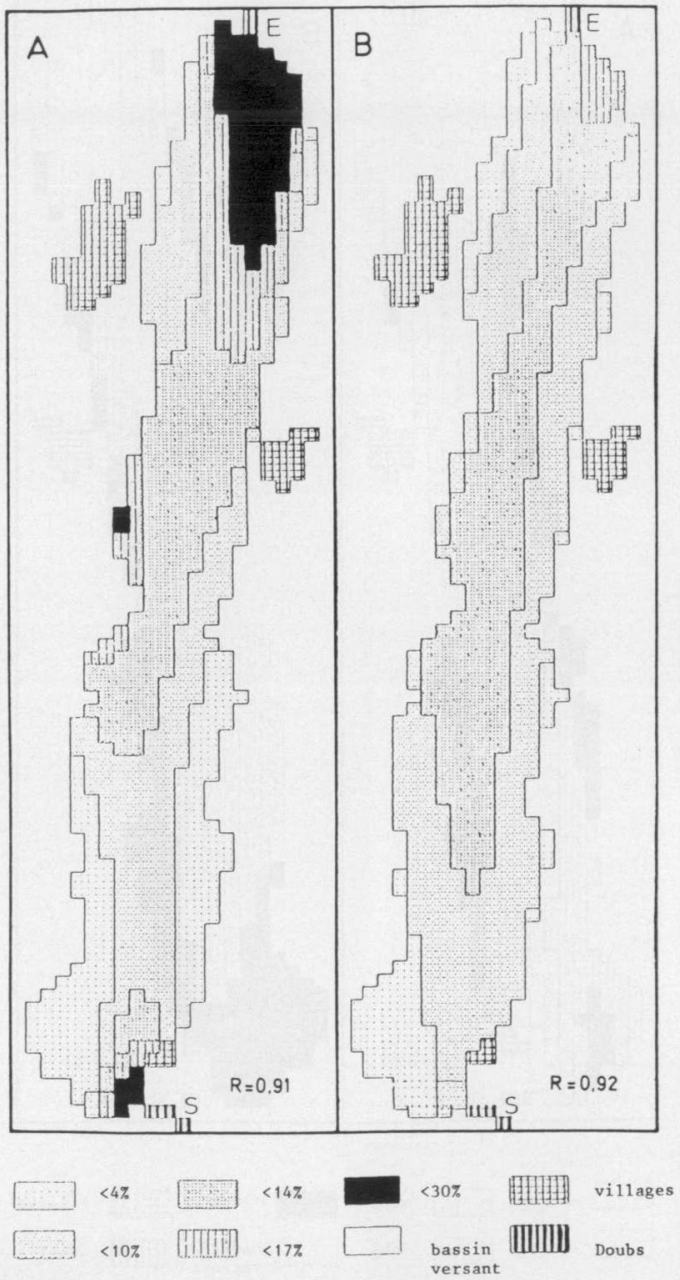

Fig. 10. Distribution spatiale de la matière organique (PF \% m.s.) dans les sédiments du lac de St Point A : strate $0-5 \mathrm{~cm}, \mathrm{~B}$ : strate $25-30 \mathrm{~cm}$. E : entrée du Doubs, S : sortie. 

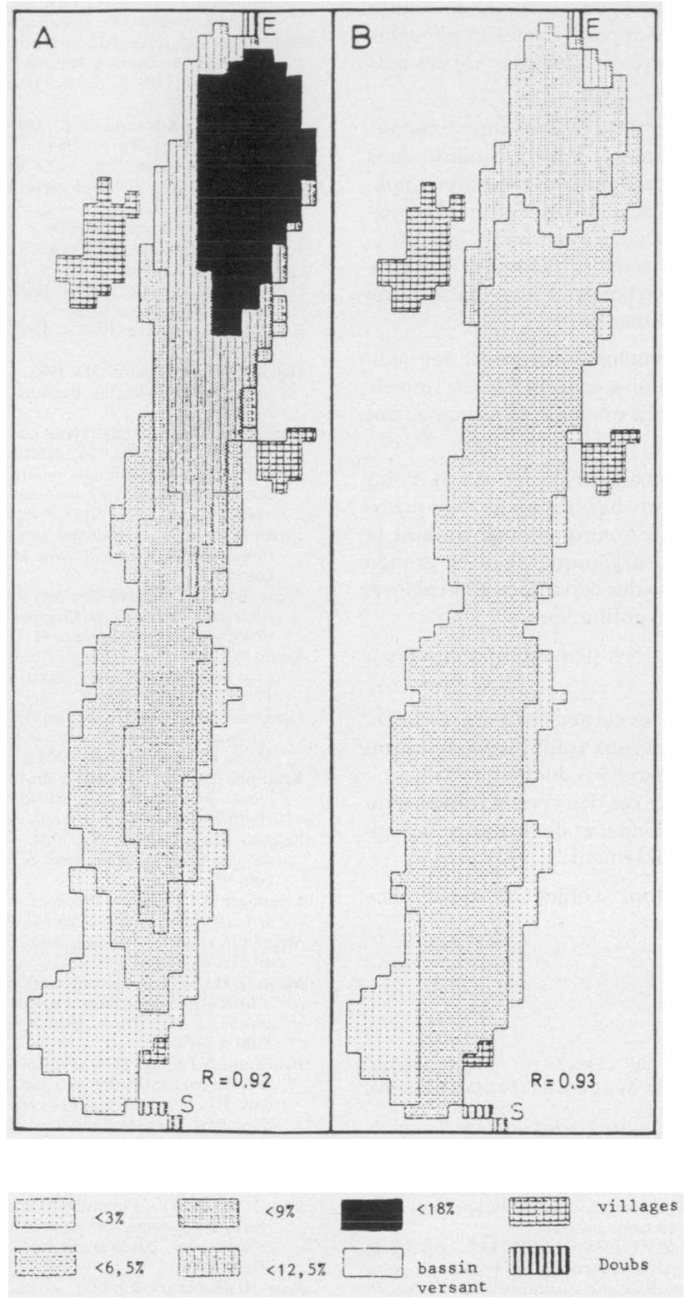

Fig. 11. Distribution spatiale de la Silice totale $\left(\mathrm{SiO}_{2} \% \mathrm{~m} . \mathrm{s}.\right)$ dans les sédiments du lac de St Point A : strate $0.5 \mathrm{~cm}, \mathrm{~B}$ : strate $25-30 \mathrm{~cm}$. E : entrée du Doubs, S: sortie. 
Doubs à la fois favorable, en assurant le renouvellement de la masse d'eau et l'oxygénation profonde. et défavorable, par ses apports azotés et phosphorés qui induisent de fortes productions algales benthiques en zone amont.

L'examen de la variabilité des résultats obtenus dans les différentes zones et strates conduit, dans le cadre d'un protocole d'analyse comparée applicable à une série de lacs, à limiter l'effort d'échantillonnage aux stations correspondant à la profondeur modale du fond lacustre (plaine) et aux strates a et c (zone de bioturbation $0-5 \mathrm{~cm}$ et $25-30 \mathrm{~cm}$ ) de chaque carotte sédimentaire.

D'un point de vuẹ typologique génèral, les sédiments apparaissent comme susceptibles de fournir une image résultante du mode de fonctionnement d'un système lacustre.

Nous tendons à considérer le lac de St Point comme l'un des plus proches, parmi les lacs jurassiens étudiés. d'un type "eufonctionnel ", dans la mesure où les apports organiques sont en grande partie minéralisés dans des conditions d'aérobiose, dominantes en zone hypolimnique.

Deux séries de relations demeurent toutefois à préciser :

- d'une part, entre les caractéristiques sédimen. taires et celles des minéraux solubilisés (en liaison avec les conditions d'oxydo-réduction) ;

- d'autre part, entre ces deux composantes d'un même ensemble fonctionnel et des critères biologiques en cours d'établissement.

Ces deux points font l'objet de recherches actuelles.

\section{Travaux cltess}

Bernard (M.) \& Deroletz (C.). 1985. - Recherches sur les sédiments de 6 lacs de Franche-Comte. Mém. D.E.S.S. Fac. Sci. Besançon, $80 \mathrm{p}$.

Blanc (C.) \& Beyne (T.). 1986. - Recherches sur les sédiments du lac de St-Point -Composantes physico-chimiques. Mèm. D.E.S.S. Fac. Sci. Besançon, $173 \mathrm{p}$

Brossard (T.) \& Joly (D.). 1987. - Plantes et Climat : pour une modelisation phénogéographique du paysage (test d'application au Svalbard). Inter-Nord, 19 (sous presse).

Brossard (T.) \& Toumeux (F.P.). 1986. - DIGICART. Logiciel de cartographie assistée par microordinateur. 4c colloque euro péen de géographie théorique et quantitative à Eindhoven. $H$. Brouillons Dupont. $14: 41-58$.

Cahill (R.A.). 1981. - Geochemistry of recent Lake Michigan sediments. III - State Geol. Surv. Circ., $517: 1-94$.
Campy (M.) \& Richard (M.). 1985. - Dynamique et typologie des remplissages lacustres tardi et post-glaciaires de la chaîne ju rassienne. Traw. Fr. Paléolimmol.. : 1-9.

Ciceri (M.F.), Marchand (B.) \& Rimberl (S.). 1977. - Introduction a tunalvse de lespace. Masson éd. Paris, $173 \mathrm{p}$.

Delebecque (A.). 1898. - Les lacs français. Chamarot et Renovard, éd., Paris, $436 \mathrm{p}$.

Domergues (Y.) \& Margenot (F.). 1970. - Ecologie microbienne du sol. Masson ed., Paris, $796 \mathrm{p}$.

E.P.R. Franche-Comté, 1982. - Le lac de l'Abbaye (massif du Jura). Etude écologique et biologique, $61 \mathrm{p}$.

E.P.R. Franche-Comté, 1984 - Les lacs de Clainaux (Ju ra). Monographies écologiques, $113 \mathrm{p}$.

E.P.R. Franche.Comté, 1985. - Les lacs Maclu (Jura). Monographies écologiques, $130 \mathrm{p}$.

E.P.R. Franche-Comté, 1986. - Le lac de Chalain (Jura). Monographies écologiques, $140 \mathrm{p}$.

E.P.R. Franche-Comté, 1981. - Le lac d'Jlay (Jura). Etude écologiqtie, $108 \mathrm{p}$

Hakanson (L.) \& Jansson (M.). 1983. - Primciples of Lake Sedimentolog, Springer-Verlag, Berlin, Heidelberg, New-York, Tokyo, $316 \mathrm{p}$.

Hansen (K.). 1961. - Lake types and lake sediments. Verh. Inter nat. Verein, Limnol, 14: 285-290.

Janel (P.). 1978. - Etude saisonniè re des formes de l'azote organi. que dans trois types d'humus sous hét raie : moder, mull acide, mull calcaire. Thèse Doct. $3^{e}$ cycle. Fac. Sci. Nancy I, 63 p.

Jarrot (I.). 1983. - Recherches sur les sédiments et la laune ben thique de quatre lacs du Jura. Mém. D.E.S.S. Fac. Sci. Besan. çon, $88 \mathrm{p}$.

Jenne (E.). 1977. - Trace elements sorption by sediments and soilsSites and processes. In Chappel (W.) \& Peterson (K.) : Molybdenum in the environment. $M$. Dekker éd. New York: 425-553.

Kemp (A.L.N.). 1971. - Organic Carbon and Nit rogen in the sur face sediments of lakes Ontario, Erié and Huron. I. Sedim. Petrol., 41 : 537-548.

Kleerekoper (Н.). 1957. - Une érude limnologique de la chimie des sédiments de tond les lacs de l'Ontario méridional. Canada. Mém. Thèse Univ. Paris, 204 p.

Krettranan (L.). 1937. - Etude hydrobiologique el aménagement pis cicole de 3 lacs du Jura utilises industriellement. Trav. Lab. Hydrobiol. Pisc. Univ. Grenoble, 27-28: 1-100.

Lagadec (B.) \& Thoumy (V.). 1984 - Recherches ecologiques sur les sediments des lacs jurassiens. Mém. D.E.S.S. Fac. Sci. Besan çon, $95 \mathrm{p}$.

Lehninger (A.L.). 1970. - Biochemistry. The molecules basis of cell structure and function. Worth. Publ, $833 \mathrm{p}$.

Magnin (A.). 1904. - La végétation des lacs du Jura. KJincksieck éd., Paris, $426 \mathrm{p}$.

Mann (K.H.). 1976. - Decomposition of marine macrophytes. In: $T$ the role of terrestrial and aquatic organisms in decomposition

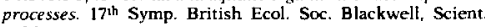
Publ. : 247-267.

Restituito (F.) \& Lair (N.). 1975-76. - Projet alpin O.C.D.E. pour la lutte contre l'eutrophisation. Lacs du Massif Central francais. III : le lac Pavin, inter-relations entre paramètres. Ann. Stat. Biol. Besse-en-Chandesse 10: 146-191.

Restituito (F.). 1984 - Contribution à l'étude des sediments d'un lac oligomésot rophe d'origine volcanique (Lac Pavin, France). Hvdrubiologia, 109 : 229-234

Rhode (W.) 1969. - Crystallization of eutrophic ation concepts in Northern Europe. In Rohlich (C.A.): Explications, causes, onsequences, correctives. Nat. Acad. Sci. Washington DC: 50.64 .

Rofes (G.) \& Savary (R.). 1981. - Description d'un nouveau modèle de carottier pour sédiments fins. Bull. Fr. Pisc., 283 : 102-113.

Spiegel (M.R.). 1982. - Théorie el applications de la statistique. Ergos et Marcotorchino ed., Paris, 358 p. 
Tipping (E.) \& Ohnstad (M.). 1984 - Aggregation of aquatic humic substances. Chemical Geol., 44 ; 349-357.

Université de Franche-Comté. 1979. - Etude écologique des lacs de St-Point et de Remoray, $114 \mathrm{p}$.

Verneaux (J.), Rémy (F.). Vidonne (A.) \& Guyard (A.). 1987. - Caractères généraux des sédiments de 10 lacs jurassiens. Sci. Eau, $6: 107-128$.
Vidonne (A.) et al. 1987. - Utilisation d'un * indice de réflectance * pour l'analyse rapide des sédiments lacustres. Sci. Eau (sous presse).

Vinogradov (A.P.). 1953. - The elementary chemical composition of marine organisms. Mem. Sears. Found Marine. Res., 2: 1-647. 